\title{
EDITORIAL
}

\section{Sperm cell biology: current perspectives and future prospects}

\author{
Asian Journal of Andrology (2011) 13, 3-5; doi:10.1038/aja.2010.155; published online 22 November 2010
}

$\mathrm{M}$ ajor advances in biomolecular techniques as well as in the sensitivity and accuracy of mass spectrometers are transforming the scientific landscape by fueling unprecedented advances in analytical biochemistry- the 'omics revolution, which refers to the study of genes (genomics), transcripts (transcriptomics), proteins (proteomics) and the various metabolites (metabolomics). It is now possible to secure inventories of lipids, proteins, metabolites and RNA species in purified cell populations and to determine how these entities change in relation to cellular function. The humble spermatozoon is an ideal target for these new technologies because these cells can be obtained in large numbers, in an absolutely pure state and can be routinely and robustly induced to perform their major biological function-fertilization-in vitro. The spermatozoon is also an excellent target for proteomics because the functional transformation of these cells during their journey from the seminiferous tubules of the testes to the surface of the oocyte, takes place in the complete absence of contemporaneous gene transcription. Rather, functionality is conferred upon these cells by post-translational changes to their protein complement that, in turn, induce critical changes in sperm metabolism. Integrating the 'omics profiles of spermatozoa holds the key to understanding the molecular mechanisms that regulate their biology. The development of these powerful new analytical tools that include DNA sequencing, DNA microarrays, mass spectrometry and protein arrays, with user interfaces that encourage their incorporation into gamete biology laboratories, will ensure that we learn more about the cell biology of mammalian spermatozoa in the next 5 years than we have learnt in the previous 50 .

In this Special Issue of Asian Journal of Andrology, we acknowledge these technical developments by highlighting contributions that have been made by some of the pioneers of sperm cell 'omics. Mark Baker, ${ }^{1}$ for example, has been responsible for developing the first detailed proteomic profiles of human, rat and mouse spermatozoa and, in so doing, has created a resource that can be used by all gamete biologists to further their research on the cell biology of spermatozoa. Mark has also pioneered the use of label free proteomics to determine how the protein structure of mammalian spermatozoa changes in concert with their physiological status during epididymal transit or capacitation. These studies have opened up new avenues of research on the roles played by membrane receptors, kinases and specific chaperones in the control of sperm function. Moreover, technologies are now available that allow us to drill down into specific subsets of proteins such as the phosphoproteome or the glycome that will further elucidate the molecular underpinnings of normal and pathological sperm function. Of course, not all aspects of sperm function are driven by changes in the proteome, and lipid biochemistry is also an extremely important facet of the regulatory process. The chapter from Joel Drevet's group ${ }^{2}$ illustrates this point beautifully. Using genetically modified mice these researchers show that the regulation of cholesterol homeostasis in the epididymis is absolutely critical in the expression of normal sperm function.

While Mark Baker ${ }^{1}$ has focused largely on the proteomic analyses of isolated spermatozoa, Xiaoyan $\mathrm{Huang}^{3}$ has concentrated on the proteomic analysis of testes and precursor germ cells, again generating inventories of proteins that will be of immense value to the entire community of scientists trying to understand the molecular regulation of male fertility. Raphael Oliva ${ }^{4}$ follows this theme by expertly summarizing the avalanche of data that is now being generated on the proteomic changes associated with spermatogenesis, epididymal maturation and capacitation, and focuses our attention on sperm chromatin. The evolution of chromatin structure is clearly a key aspect of sperm cell biology. It begins with chromatin remodeling during spermiogenesis to create a highly compacted, crosslinked nucleus that is absolutely unique to this cell type and extends to the subsequent unraveling of this structure in the oocyte, following fertilization.

The mechanisms that control the remodeling of sperm chromatin during spermiogenesis and epididymal maturation are clearly extremely complex and are only now being resolved. Steven Ward is an international authority on this topic and has prepared an authoritative summary ${ }^{5}$ of current concepts and, specifically, emerging evidence suggesting an important role for the nuclear matrix in the functional organization of sperm chromatin. According to this model, points of attachment between the DNA linker regions and the nuclear matrix may be important for the initiation of DNA cleavage during apoptosis by topoisomerase II. It would indeed be interesting to know whether the factors that induce apoptosis in human spermatozoa consistently generate $50-\mathrm{kb}$ fragments typical of nuclear toroids. Additionally, DNA can also be damaged by reactive oxygen species (ROS) that induce oxidized base adduct formation (largely 8-hydroxy-2'-deoxyguanosine), leading to the creation of abasic sites, destabilization of the DNA backbone and fragmentation. In this case, however, the DNA fragmentation will not be into discrete $50-\mathrm{kb}$ toroid fragments, but into much smaller fragments in the $20-25 \mathrm{~kb}$ range. ${ }^{6}$ In the review of DNA damage, John Aitken ${ }^{7}$ points out the possible involvement of ROS generated during sperm apoptosis in the etiology of defective sperm function and DNA damage. Ralf $\mathrm{Henkel}^{8}$ also provides a very comprehensive discussion of the importance of oxidative stress in the impairment of sperm function and the chemical principles that underpin these negative effects. In this chapter, the nature of the ROS source 
is shown to have a significant impact on the degrees of defective sperm function and DNA damage observed. The most common intracellular origin of ROS appears to be aberrant electron transfer through the mitochondrial electron transport chain, while leukocytes comprise the most significant extracellular source of toxic oxygen metabolites. Managing the oxidative stress originating from these sources has to be conducted with care because the cellular mechanisms involved in the physiological regulation of sperm function are also redox-sensitive. In some circumstances, peroxidation (unlike Caesar) should be praised not buried, so the blanket use of antioxidants to smother free radical generation in the germ line should be carefully considered as excessive scavenging of ROS might lead to sperm dysfunction.

In animals models there is incontrovertible evidence that the experimental induction DNA damage in the male germ line has dramatic effects on the subsequent progress of pregnancy and the health and wellbeing of the offspring. ${ }^{9}$ How effectively these data translate to the clinical situation is controversial, but the complexities of this field have been elegantly described by Mona Bungum. ${ }^{10}$ DNA damage assays such as the sperm chromatin structure assay have been shown to be independent predictors of pregnancy in first-pregnancy planners as well as in couples undergoing intrauterine insemination. However, the data are more conflicting when consideration is given to the relationships between DNA damage and fertilization, pre-embryo development and pregnancy outcome during in vitro fertilization or intracytoplasmic sperm injection. Clearly in our species, clinical outcomes are modulated by a variety of confounding factors, including the nature of the insemination process and the capacity of the oocyte for DNA repair, which obfuscate the relationships that the animal data tell us must be there.

In addition to its significance as a target for DNA damage, sperm chromatin structure, particularly the nuclear matrix, may be important for orchestrating the replication of DNA in the zygote and thus the initiation of early embryonic development. Hence, it is becoming abundantly clear that a variety of epigenetic factors associated with the male germ line are critical for the establishment of normal embryonic development. In their authoritative reviews of this important subject, both Douglas Carrell ${ }^{11}$ and Steven Ward ${ }^{5}$ emphasize the importance of DNA methylation status, histone modifications and protamination in establishing a genome that will: (i) be stabilized for the perilous journey through the male and female reproductive tracts and yet (ii) be able to decondense within the oocyte, replicate, and ultimately initiate a pattern of gene expression commensurate with normal embryogenesis. Of particular interest is Carrell's discovery that the epigenetic programming of the spermatozoon's genome places certain genes in a 'poised' state, ready for activation during early embryonic development.

In terms of the gamete itself, this Special Issue contains a number of insightful, erudite reviews that bring us up to date with the current status of this field. The power of functional genomics as a tool for unraveling the molecular mechanisms regulating sperm function is expertly presented by a pioneer in this field, Masaru Okabe. ${ }^{12}$ This chapter highlights the fact that a number of knockouts have been generated (Calmegin, Adam1a, Adam2, Adam3, Ace and Pgap1) that disrupt the fertilizing potential of mouse spermatozoa rather than their production. Intriguingly, all of these genetically modified mice have defects in zona-binding coupled with an inability to migrate to the site of fertilization in vivo. Functional genomics has forced us to reappraise the whole process of sperm-zona recognition. It is now clear that the lock-and-key mechanisms that were originally proposed to mediate this binding event are a gross oversimplification of the actual process. Andrew Reid and colleagues ${ }^{13}$ have advanced an alternative hypothesis to explain this process that is based on the assembly of a multireceptor zona recognition complex on the sperm surface during capacitation. The assembly of such complexes is driven by the phosphorylation of molecular chaperones that assemble and insert these assemblages into 'lipid rafts'. This hypothesis explains why the specific deletion of candidate zona receptor molecules, such as GalT, has failed to generate the anticipated loss of zona binding potential. It is because there is an inherent redundancy in this recognition system, which is designed as a fail-safe mechanism to ensure that sperm-egg recognition is successfully achieved.

Of course, sperm binding to the zona pellucida is just the first step in an elaborate cascade of cellular interactions involving the male and female gametes that culminate in fertilization. Satish Gupta and his team ${ }^{14}$ summarize our current understanding of zona pellucida composition including their exciting studies on the way in which the zona glycoproteins interact with the sperm surface to stimulate the acrosome reaction. The nature of this interaction between the sperm surface and the zona pellucida and the nature of the signal transduction pathways activated as a result of this interaction remain one of the central conundrums in sperm cell biology. The area is full of interesting, challenging hypotheses and it is truly exciting to see how rapidly this field is evolving as the underlying mechanisms yield to the power of functional genomics and protein biochemistry.

The preparation of spermatozoa for binding to the zona pellucida and undergoing the acrosome reaction is dependent on these cells attaining a capacitated state. The latter has been recognized as a biological phenomenon since the 1950s but it only now that we are beginning to understand the molecular mechanisms that underpin this process. Haim Breitbart ${ }^{15}$ has made important contributions to our understanding of sperm capacitation over many years and his review addresses the important role of epidermal growth factor receptor in these processes including novel findings on the transactivation of this receptor by protein kinase A, ouabain and G protein-coupled receptors. Finally Moira O'Bryan's team ${ }^{16}$ provides an excellent example of the new generation of talented scientists that are employing the tools of the 'omics revolution to unravel the mysteries of sperm differentiation and function and, in their case, the potential role that the Crisp family of proteins might play in this process.

Overall, this Special Issue of Asian Journal of Andrology gives an important authoritative snapshot of current thinking in sperm cell biology. We commend the contents to any researcher or clinician interested in following the exponential growth in our knowledge of the molecular mechanisms regulating the pathology and physiology of these endlessly intriguing cells.

(Guest editors of this issue) R John Aitken ${ }^{1}$ and Ralf R Henkel ${ }^{2}$ ${ }^{1}$ Discipline of Biological Sciences, University of Newcastle, Newcastle, NSW 2308, Australia (jaitken@mail.newcastle.edu.au) and ${ }^{2}$ Department of Medical Biosciences, University of the Western Cape, Bellville 7535, South Africa (rhenkel@uwc.ac.za)

1 Baker MA. The 'omics revolution and our understanding of sperm cell biology. Asian J Androl 2011; 13: 6-10.

2 Saez1 F, Ouvrier A, Drevet JR. Epididymis cholesterol homeostasis and sperm fertilizing ability. Asian J Androl 2011; 13: 11-17.

3 Huang XY, Sha JH. Proteomics of spermatogenesis: from protein lists to understanding the regulation of male fertility and infertility. Asian J Androl 2011; 13: 18-23.

4 Oliva R, Castillo J. Proteomics and the genetics of sperm chromatin condensation. Asian J Androl 2011; 13: 24-30. 
5 Yamauchi Y, Shaman JA, Ward WS. Nongenetic contributions of the sperm nucleus to embryonic development. Asian J Androl 2011; 13: 31-35.

6 Sawyer DE, Mercer BG, Wiklendt AM, Aitken RJ. Quantitative analysis of gene-specific DNA damage in human spermatozoa. Mutat Res 2003; 529: 21-34.

7 Aitken RJ, Koppers AJ. Apoptosis and DNA damage in human spermatozoa. Asian J Androl 2011; 13: 36-42.

8 Henkel RH. Leukocytes and oxidative stress: dilemma for sperm function and male fertility. Asian J Androl 2011; 13: 43-52.

9 Aitken RJ, de luliis GN. On the possible origins of DNA damage in human spermatozoa. Mol Hum Reprod 2010; 16: 3-13.

10 Bungum M, Bungum L, Giwercman A. Sperm chromatin structure assay (SCSA): a tool in diagnosis and treatment of infertility. Asian J Androl 2011; 13: 69-75.
11 Jenkins TG, Carrell DT. The paternal epigenome and embryogenesis: poising mechanisms for development. Asian J Androl 2011; 13: 76-80.

12 Inoue N, Ikawa M, Okabe M. The mechanism of sperm-egg interaction and the involvement of IZUMO1 in fusion. Asian J Androl 2011; 13: 81-87.

13 Reid AT, Redgrove K, Aitken RJ, Nixon B. Cellular mechanisms regulating sperm-zona pellucida interaction. Asian J Androl 2011; 13: 88-96.

14 Gupta SK, Bhandari B. Acrosome reaction: relevance of zona pellucida glycoproteins. Asian J Androl 2011; 13: 97-105.

15 Breitbart $\mathrm{H}$, Etkovitz $\mathrm{N}$. Role and regulation of EGFR in actin remodeling in sperm capacitation and the acrosome reaction. Asian J Androl 2011; 13: 106-110.

16 Koppers AJ, Reddy T, O'Bryan MK. The role of cysteine-rich secretory proteins in male fertility. Asian J Androl 2011; 13: 111-117. 